\title{
Analyses of mode coupling in joined parallel phononic crystal waveguides
}

\author{
Jia-Hong Sun and Tsung-Tsong Wu \\ Ultrasonics Laboratory, Institute of Applied Mechanics, National Taiwan University, Taipei 106, Taiwan \\ (Received 1 September 2004; revised manuscript received 25 February 2005; published 24 May 2005)
}

\begin{abstract}
In this paper, we present an analysis of coupling effects in joined parallel phononic crystal waveguides. The finite difference time domain (FDTD) method with periodic boundary condition is adopted to analyze the band gaps and dispersion relation of phononic waveguides. The defect modes of a single phononic waveguide are analyzed and first discussed to serve as a basis for a joined waveguides system. Then, the dispersion relation and displacement field of supermodes of joined waveguides are calculated and discussed. Both displacement pattern and transmission coefficient of the defect modes are calculated. To transfer the power from one waveguide to another, the coupling lengths are evaluated by numerical experiments and can be understood by the concept of beat length. Finally, we analyze an elastic waveguide coupler and demonstrate that the coupler can potentially be employed as a power switch of the acoustic wave.
\end{abstract}

DOI: 10.1103/PhysRevB.71.174303

PACS number(s): 63.20.-e, 43.20.+g, 43.40.+s, 46.40.Cd

\section{INTRODUCTION}

In recent years, much research has been devoted to photonic crystals that demonstrate the band gaps of electromagnetic (EM) wave propagation in periodic dielectric media. Due to the analogy between elastic waves and electromagnetic waves, inhomogeneous media consisting of different elastic materials have also become a topic of academic interest. Such inhomogeneous structures, named "phononic crystals," typically consist of two or more materials with a periodic arrangement. The acoustic band gap (ABG) is similar to the optic band gap in photonic crystals in that the acoustic wave propagation in a certain frequency range is forbidden. ${ }^{1-3}$ In addition to bulk acoustic waves, band gaps for surface acoustic waves have also been demonstrated. ${ }^{4-6}$ The existence of large acoustic band gaps has been revealed by several phononic crystal examples made of solid or fluid constituents. $^{7-11}$

The total band gap of elastic wave propagation toward any direction in the phononic crystals has been predicted and observed. ${ }^{12}$ The localization of elastic waves with the arrangement of defects in phononic crystals as a linear waveguide was also studied through the calculation of the transmission coefficient. ${ }^{13}$ The research of waveguide structures such as linear straight waveguides with resonant cavities or with a vertical stub was reported. ${ }^{14-17}$ Alternative inclusions of phononic crystals were also discussed, and a narrow pass band was designed in a liquid-based phononic crystal structure. ${ }^{18,19}$ These studies inspired the research of coupling behavior of joined parallel linear phononic crystal waveguides in this paper.

When one linear waveguide is close enough to another in a phononic crystal structure, the coupling effect between the two waveguides is so strong that they are bounded as a single system. Each eigenvalue corresponding to an eigenstate of the single waveguide splits into two values in the case of the joined waveguides system. This exciting phenomenon is similar to what appeared in photonic crystals. ${ }^{20-22}$

The calculations in our work are based on the finite difference time domain (FDTD) method. The defect mode of elastic wave propagation is analyzed with the periodic boundary condition by applying the Bloch theorem. The supermodes of joined parallel phononic crystal waveguides are studied so that the beat phenomenon is revealed to evaluate the coupling lengths of the system of joined waveguides. Therefore, an elastic wave coupler is demonstrated and, further, an enhanced coupler is presented. Due to a stronger coupling effect, the enhanced coupler shortens the coupling length and reduces the size of the coupler.

To make our arguments clear, the method of calculation and defect modes of a single waveguide in the phononic crystals are introduced in Sec. II. The coupling effect of joined parallel waveguides is reported in Sec. III. Later, the dispersion relation and displacement field of supermodes for the coupling waveguides are discussed. The coupling length in a system of two joined waveguides is also introduced. The analysis of an elastic coupler in the system of two waveguides is in Sec. IV. Lastly, a summary of the results is presented in Sec. V.

\section{DEFECT MODES OF A PHONONIC CRYSTAL WAVEGUIDE}

In previous research, the acoustic band gap of elastic wave propagation in phononic crystals was identified by calculating the dispersion curves or the transmission coefficient. The most popular technique employed to analyze the dispersion relation of elastic waves in phononic crystals is the plane wave expansion (PWE) method. The PWE method transforms the elastic wave equation into Fourier space and solves the simplified eigenvalue problem. With the arrangement of defects, the straight linear waveguide can still be analyzed by the PWE method with the concept of a supercell. However, to solve the eigenvalue problem of PWE with the supercell condition, it takes a great number of wave vectors in the reciprocal space to guarantee an accurate value of modes and limits the efficiency of calculation.

Generally, the three-dimensional heterogeneous finite difference formulation with staggered grids is adopted to study the wave propagation in phononic crystals. The composite structures of phononic crystals can be easily calculated by 


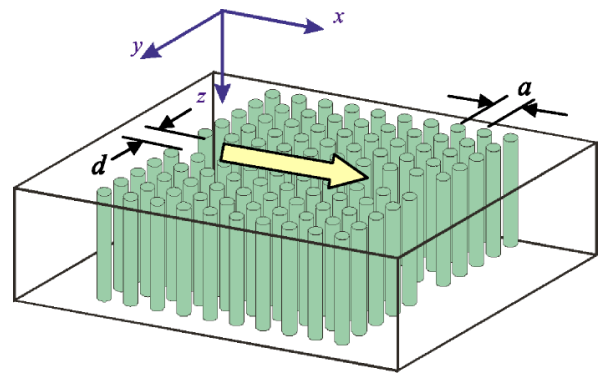

FIG. 1. The oblique view of a two-dimensional phononic crystal waveguide. The lattice constant $a$ and the width of waveguide $d$ are marked in the figure.

replacing the material constant of the corresponding location. Tanaka et al. ${ }^{23}$ first introduced the Bloch theorem to treat the periodic boundary condition of the unit cell of phononic crystals. By recording the displacement inside the unit cell and taking the Fourier transformation, the eigenfrequencies of a given wave vector $\boldsymbol{k}$ are indicated by selecting the resonance peaks of the spectrum. The advantages of adopting the FDTD method to calculate dispersion curves include less calculation time, more material-selecting flexibility, and inclusion shape variation. Due to the discrete grids, one possible error is the approximate shape of inclusions. In this paper, the FDTD method is employed to solve the supercell problem for obtaining dispersion relations of single waveguide defect modes and joined waveguides supermodes. To be more efficient, the parallelism computation program with a message passing interface (MPI) is realized and the calculations are executed on a PC cluster system with 16 CPUs.

The phononic crystal, which we take into consideration, is a solid/solid two-dimensional phononic crystal consisting of epoxy as the base and steel as the cylinders. The lattice constant $a$ of the phononic crystal structure is $8 \mathrm{~mm}$, and the radius of the cylinder is $3 \mathrm{~mm}$. In this case, the filling ratio $f$ is equal to 0.442 . To simulate the wave propagation in the structure using the FDTD method, each unit cell is divided into $24 \times 24$ grids, with a time step interval of $20 \mathrm{~ns}$. The density and elastic constant $\mathrm{C}_{11}$ and $\mathrm{C}_{44}$ of steel are assumed as $7900 \mathrm{~kg} / \mathrm{m}^{3}, 280.2 \mathrm{GPa}$, and $82.9 \mathrm{GPa}$, respectively, and those for epoxy are $1180 \mathrm{~kg} / \mathrm{m}^{3}, 7.61 \mathrm{GPa}$, and $1.59 \mathrm{GPa}$. In the two-dimensional phononic crystal cases, as shown in Fig. 1 , the elastic wave propagates within the $X-Y$ plane perpendicular to the cylinders and the phononic structure is infinite along the $Z$ direction. Thus, the polarization modes of elastic waves can be decoupled into the in-plane mode and the antiplane mode $(X-Y$ plane). The present work is to study phenomena of waveguides, and therefore we consider the inplane mode wave propagation, which includes longitudinal and transverse waves.

Dispersion curves of steel/epoxy phononic crystal are calculated first. The displacement is recorded within 200000 time steps and the Fourier transform is applied to find the resonance frequencies. Our result shows that the total band gap of this phononic crystal is located between $86 \mathrm{kHz}$ and $197 \mathrm{kHz}$. By applying the concept of a supercell, dispersion curves of a linear straight waveguide in phononic crystals can be calculated by combining several basic unit cells. The
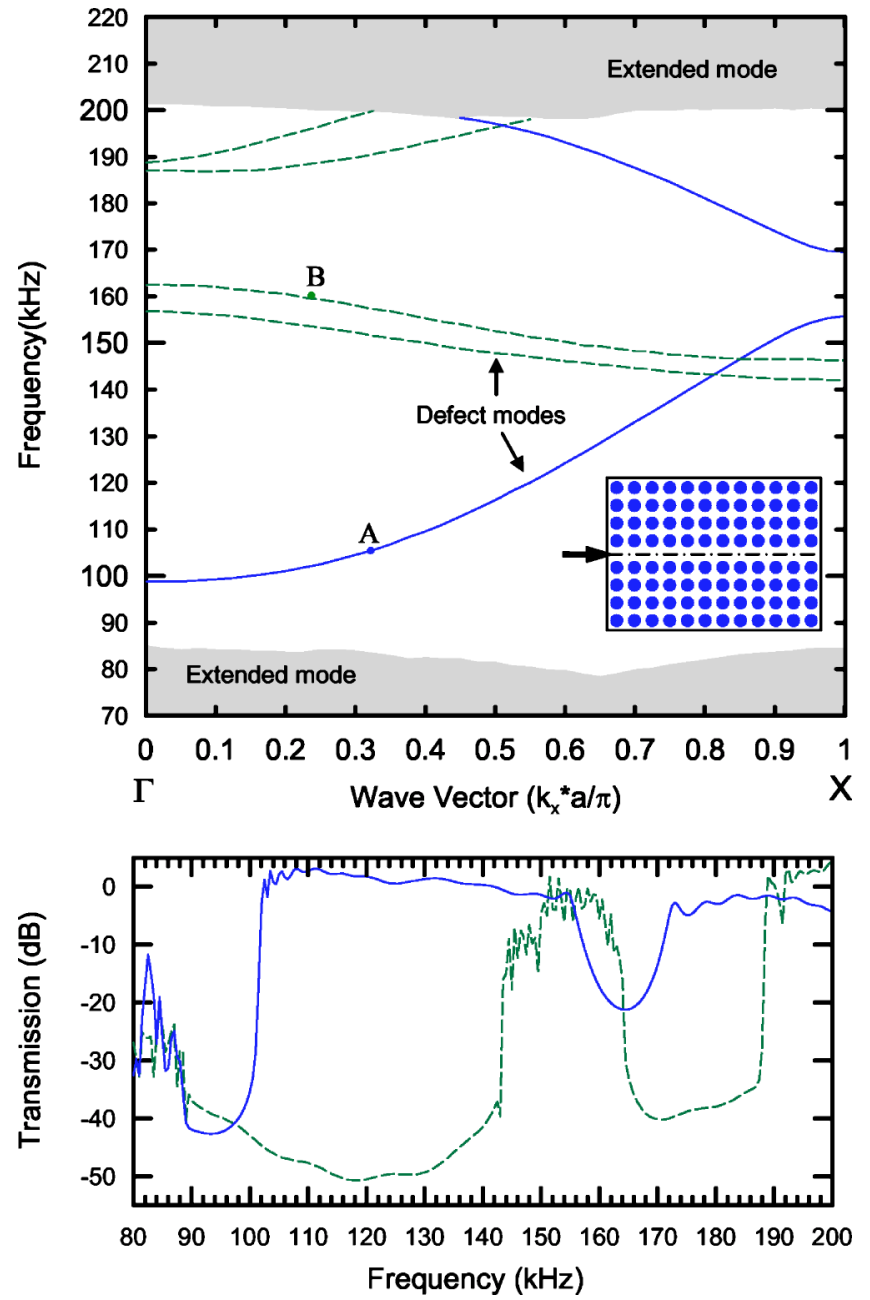

FIG. 2. (Color online) Defect modes of the elastic wave inside the phononic crystal waveguide. (a) Dispersion curves of defect modes. (b) The transmission coefficient through the waveguide.

elastic wave mostly propagates within the waveguide, but also leaks slightly to the neighboring area. The allowed modes inside the waveguide include the extended modes, which fall outside the total band gap, and defect modes, which fall in the range of total band gaps. The results are consistent with those reported by Khelif et al. ${ }^{15}$

To investigate the coupling effect of waveguides, we consider a supercell consisting of $8.5 \times 1$ unit cells and a waveguide with a $6 \mathrm{~mm}$ width (the distance between two neighboring cylinders on both sides). The inset of Fig. 2(a) shows the cross section of the single waveguide, and a dasheddotted line denotes the mirror plane of the structure. Similar to the conventional acoustic waveguides, the defect modes in Fig. 2(a) can be classified as symmetric modes (solid lines) and antisymmetric modes (dashed lines), with respect to the mirror plane of the waveguide. In the range of total band gap, the symmetric modes extend in the frequency intervals 99-156 and $169-198 \mathrm{kHz}$, and the antisymmetric modes extend in intervals $142-163$ and $187-198 \mathrm{kHz}$.

The defect modes of the waveguide are identified by the transmission coefficients and the displacement fields. First, we launch a Gaussian weighted plane-wave package with a 
(a) $\underline{105 \mathrm{kHz}}$
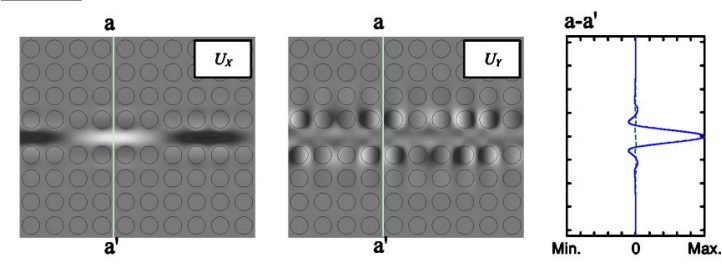

(b) $160 \mathrm{kHz}$
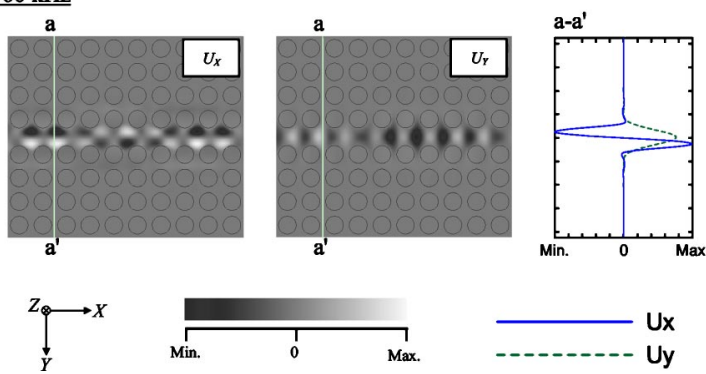

FIG. 3. Elastic wave displacement fields inside the phononic crystal waveguide. (a) The displacement fields of the $105 \mathrm{kHz}$ longitudinal wave which belongs to the symmetric mode. (b) The displacement fields of the $160 \mathrm{kHz}$ transverse wave which belongs to the antisymmetric mode.

central frequency of $140 \mathrm{kHz}$ and with a duration of $100 \mu \mathrm{s}$. The transmission coefficient is obtained by taking a Fourier transform of the average of elastic wave displacements recorded across the waveguide and comparing the spectra with those without phononic crystals. Since the polarization of a longitudinal wave is along the $X$ direction, the longitudinal wave excites the symmetric modes. Likewise, polarization of the transverse wave is along the $Y$ direction, therefore inducing antisymmetric modes inside the single waveguide. Figure 2(b) shows that the transmission coefficient of the elastic wave propagating inside the waveguide is a function of frequency. In our example, the studied waveguide is 15 lattice constants in length, which, accordingly, lets the transmission coefficient fully reflect the properties of the band gap. For the symmetric modes, a gap exists below $102 \mathrm{kHz}$ and another gap is in the interval $155-172 \mathrm{kHz}$, while the pass bands for antisymmetric modes are between 144 and $164 \mathrm{kHz}$ and those higher than $188 \mathrm{kHz}$. Indeed, the defect modes are clearly identified by the transmission coefficients.

Next, we apply the FDTD approach to calculate the displacement fields of elastic waves. We consider the phononic crystal structure containing $8.5 \times 10$ unit cells. The periodic condition is used along the boundaries in the $Z$ direction for the two-dimensional structure. In addition, the perfectly matched layer (PML) ${ }^{24,25}$ is adopted as the absorbing boundary condition for the boundaries in the $X$ and $Y$ directions. The longitudinal wave, with a specific central frequency $f$, is generated to excite symmetric defect modes, while the transverse wave is stimulated for antisymmetric defect modes.

In Fig. 3(a), we show the displacement fields of point A marked in Fig. 2(a). The symmetric mode is excited by the longitudinal wave with $105 \mathrm{kHz} . U_{X}$ and $U_{Y}$ represent the displacement components along the $X$ and $Y$ directions; their values of the cross section are shown in the gray scale. The white color and black color represent the maximum value and minimum value, respectively. For better understanding, we also plot the displacement components along $a-a^{\prime}$ to identify the modes with a solid line representing $U_{X}$ and a dashed line representing $U_{Y}$. Obviously, the displacement components in both directions are symmetric to the mirror plane of the waveguide. Meanwhile, Fig. 3(b) shows the result of point B in Fig. 2(a) with the $160 \mathrm{kHz}$ transverse wave, which belongs to the antisymmetric modes. We note that the displacement fields of the chosen points A and B in Fig. 2(a) demonstrate the symmetric and antisymmetric modes, thereby coinciding with the transmission analyses shown in this section.

\section{COUPLINGS OF PHONONIC CRYSTAL WAVEGUIDES}

The manipulation of defects in photonic crystals induced several optic communication applications, including the low loss transmission bending structures and wavelength division multiplexing (WDM) devices. ${ }^{20}$ The analogy of photonic crystals and phononic crystals inspires the research of coupling phononic crystal waveguides. In the following, we present analyses of joined parallel phononic crystal waveguides.

\section{A. Coupling effect and supermodes of coupling phononic waveguides}

Waveguide in a phononic crystal structure can be considered as a composition of adjacent point defects. Thus, the individual modes of point-defect unit cells will couple as the defect modes of a waveguide. Similarly, two waveguides will join together as a single system when one linear waveguide is close enough to another. The eigenvalue corresponding to eigenstates of the single waveguide will split into different modes, which results in a coupling effect between these two joined waveguides. Dispersion curves of coupling modes of the system can be calculated as those in the single waveguide using the FDTD method. We consider a system of two linear straight waveguides with the width of each waveguide being $6 \mathrm{~mm}$, coincidentally the same as the previous case in Sec. II. In this case, there is one row of phononic unit cells between the two waveguides, as shown in the inset of Fig. 4(a). The two nearby waveguides can be considered as one joined system which supports the supermodes, i.e., the allowed defect modes of the system. They can also be obtained by the dispersion relation.

The supermodes of the system of joined waveguides are calculated with a supercell consisting of $10 \times 1$ unit cells. Figure 4(a) demonstrates the results of dispersion curves. The solid lines are the supermodes of the coupling waveguides system and the dashed lines are the defect modes of a single waveguide system, as shown in Fig. 2(a). The dispersion curves of the system of two waveguides appear in pairs due to the coupling effects. Thus, it seems that the supermodes are split from defect modes of the single waveguide. In this phononic crystal structure, if the number of rows of phononic unit cells between two waveguides is larger than two, then the coupling effect is hard to detect. Apparently, the coupling effect of waveguides depends on the distance between two waveguides. 

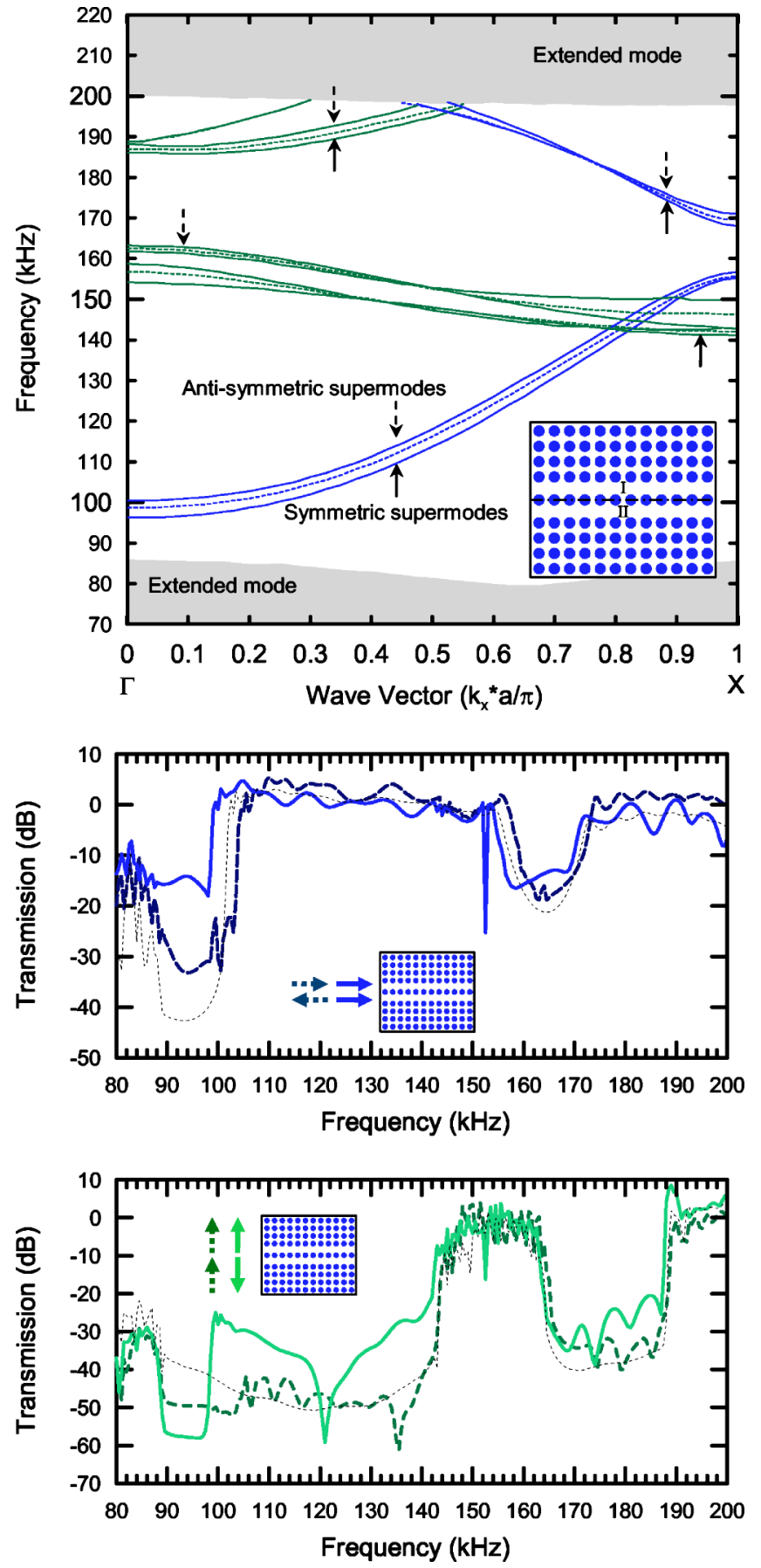

FIG. 4. (Color online) The supermodes of the system of joined phononic crystal waveguides. (a) The dispersion curves of the supermodes. Solid arrows indicate the symmetric supermode and dashed arrows indicate the antisymmetric supermodes. (b) Transmission coefficients of symmetric and antisymmetric longitudinal wave cases. (c) Transmission coefficients of symmetric and antisymmetric transverse wave cases.

It can be understood that for two identical waveguides, the lowest-order modes include even (symmetric) and odd (antisymmetric) polarity with respect to the mirror plane between the waveguides. In this case, the cross section of the two joined waveguides is shown in the inset of Fig. 4(a), with a dashed-dotted line representing the mirror plane.
Symmetric and antisymmetric types of supermodes are marked in Fig. 4(a). Solid arrows indicate symmetric supermodes and dashed arrows indicate antisymmetric supermodes. Similarly, the types of supermodes are examined in two ways: the analysis of transmission coefficients and displacement fields.

Transmission coefficient is calculated by comparing the spectra of penetrating waves, as we mentioned in Sec. II. To examine the mode type of a system of two joined waveguides, the incident waves include both the longitudinal wave and the transverse wave. Unlike the symmetric mode, which is only excited by longitudinal waves in a single waveguide, both symmetric and antisymmetric modes can be excited by longitudinal waves in the system of joined waveguides. Symmetric and antisymmetric cases are controlled by modulating the phase of incident waves. The symmetric (antisymmetric) case of longitudinal waves means the incident longitudinal wave is symmetric (antisymmetric) with respect to the mirror plane. The transmissions are shown in Fig. 4(b). A solid line shows the symmetric supermode while a dashed line shows the result of the antisymmetric supermode. In comparison with the joined parallel waveguides, the result of the longitudinal wave inside a single waveguide in Fig. 2(b) is also plotted as a dotted line. We find that the frequency range of the symmetric supermode shifts toward lower frequency while the range of the antisymmetric supermode shifts toward higher frequency. This phenomenon helps make the supermodes clear in Fig. 4(a). Symmetric and antisymmetric cases of transverse waves are also calculated, and transmissions of both cases are shown in Fig. 4(c). The definition of the lines is the same as those of Fig. 4(b). The frequency ranges of the supermodes also have a similar tendency to that of the longitudinal wave.

The chosen displacement fields in Fig. 5 show propagation modes of the elastic wave. The setup of the numerical calculation is similar to the examples of Fig. 3, except that the structure is composed of $10 \times 10$ unit cells. In this example, elastic waves of the central frequency $f=110 \mathrm{kHz}$ propagating inside the system of two joined waveguides are demonstrated. The longitudinal waves are excited at these two waveguide entrances with the symmetric supermode in the synchronous phase and the antisymmetric supermode in the inverse phase, both with respect to the mirror plane. The displacement fields are shown in Figs. 5(a) and 5(b). The definition of the color and lines in Fig. 5 is the same as that in Fig. 3. Since the incident waves are polarized along the $X$ direction, the displacement component $U_{X}$ is significantly larger than $U_{Y}$. The results in Fig. 5(a) clearly demonstrate the symmetry of both $U_{X}$ and $U_{Y}$. On the other hand, the results in Fig. 5(b) show the antisymmetric property. The wavelength is evaluated by lattice constant $a$, and the values are 5.9a and 4.7a in Figs. 5(a) and 5(b), respectively. It reveals that wave vector $\boldsymbol{k}$ of the symmetric supermode is larger than that of the antisymmetric supermode for waves with the same central frequency. This result is also in good agreement with the modes indicated in Fig. 4(a).

\section{B. Coupling length of coupling waveguides}

Supermode dispersion of a system of joined parallel waveguides implies that if there is a constant frequency 
(a) $110 \mathrm{kHz}$, anti-symmetric mode
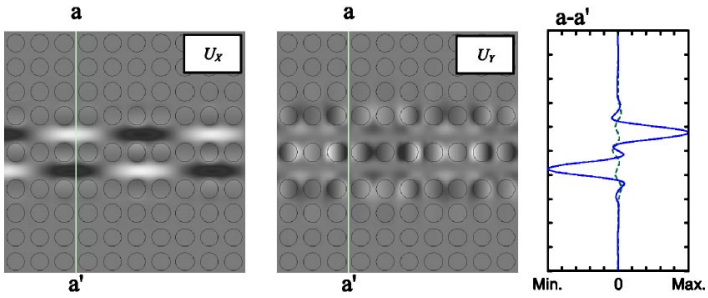

(b) $110 \mathrm{kHz}$, symmetric mode
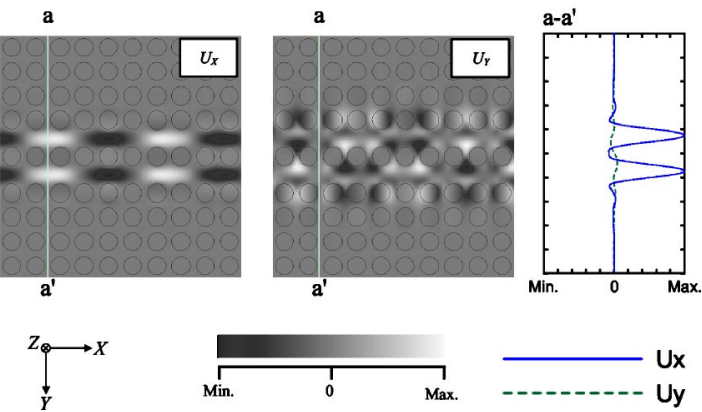

FIG. 5. Displacement fields of the elastic wave propagation inside the phononic crystal waveguide. (a) The $110 \mathrm{kHz}$ antisymmetric supermode elastic wave. (b) The $110 \mathrm{kHz}$ symmetric supermode elastic wave.

source inside the waveguide, then it is possible that there are more than two allowed modes with different wave vectors $\boldsymbol{k}$. Hence, the beat phenomenon performs in the system of joined parallel waveguides if both symmetric and antisymmetric supermodes arise. The following study reveals the beat phenomenon due to the coupling effect of joined waveguides.

Numerical experiments of wave propagation in the system of two joined waveguides are conducted by using the FDTD method. To investigate the mode coupling effect, we employ a simple linear two-waveguide structure which has one row of unit cells between the waveguides as shown in Fig. 6(a). In Fig. 6(b), an elastic wave with $f=110 \mathrm{kHz}$ propagating into the upper waveguide from the left entrance will transfer back and forth between the lower and upper waveguides. A similar result is also observed for the elastic wave with $f$ $=140 \mathrm{kHz}$ in Fig. 6(c). The necessary distance for the elastic wave to transfer from one waveguide to another is called the coupling length. It can be observed that the coupling length of the elastic wave of $f=110 \mathrm{kHz}$ is smaller than the one of $f=140 \mathrm{kHz}$, whose values are $11.7 a$ and $22.5 a$, respectively. In Fig. 6(d), the elastic wave of $f=155 \mathrm{kHz}$ also transfers between the two waveguides with the coupling length being about 25.1 $a$. However, since there are more than two allowed modes of the elastic wave, the energy transformation pattern between the two waveguides is more complicated than the cases of $f=110$ and $140 \mathrm{kHz}$. We choose the frequency range of $105-145 \mathrm{kHz}$ for later calculations because of the simple split-modes condition. FDTD numerical experiments conclude the correlation curve of coupling length and elastic wave frequency as the solid line in Fig. 7, where the coupling length varies from $7.9 a$ to $22.5 a$ in the range $105-145 \mathrm{kHz}$. (a)

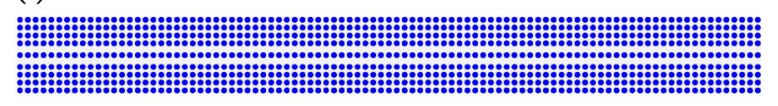

(b) $110 \mathrm{kHz}$

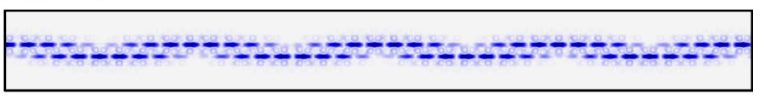

(c) $140 \mathrm{kHz}$

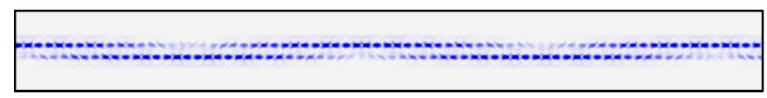

(d) $155 \mathrm{kHz}$

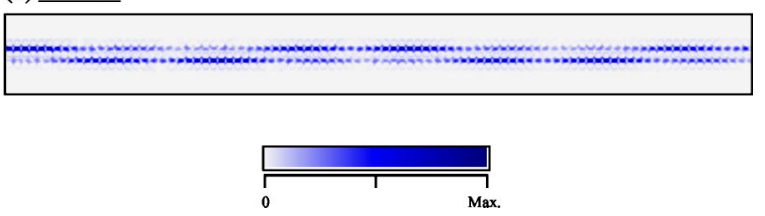

FIG. 6. Amplitude fields which show power transfer between joined parallel phononic crystal waveguides. (a) The cross section of the system of joined waveguides. (b) The amplitude field for the $110 \mathrm{kHz}$ incident wave. (c) The amplitude field for the $140 \mathrm{kHz}$ incident wave. (d) The amplitude field for the $155 \mathrm{kHz}$ incident wave.

We note that the coupling length can be analyzed with the knowledge of beat frequency in traditional optics. ${ }^{26}$ Beat phenomena between the symmetric and antisymmetric modes arise when excitation is neither symmetric nor antisymmetric. Since the corresponding wave vectors $\boldsymbol{k}$ of the symmetric and antisymmetric supermodes are different when the elastic wave has the same frequency, the relative phase of these modes will shift when the wave propagates. Therefore, the coupling length is estimated as half a beat length of the two supermodes and can be derived from the dispersion curves. Beat length $L_{B}$ is computed as $L_{B}=2 \pi /\left|k_{S}-k_{A}\right|$, where $k_{S}$ and $k_{A}$ are the corresponding symmetric and antisymmetric wave vectors of the chosen frequency and can be decided from supermode dispersion curves. For example, the corresponding normalized wave vectors are 0.446 and 0.379 for the elastic wave of $f=110 \mathrm{kHz}$ in Fig. 4. Thus, the beat length can be evaluated as $L_{B}=2 a /|0.446-0.379| \approx 29.85 a$ and the coupling length is approximately $14.9 a$. The pre-

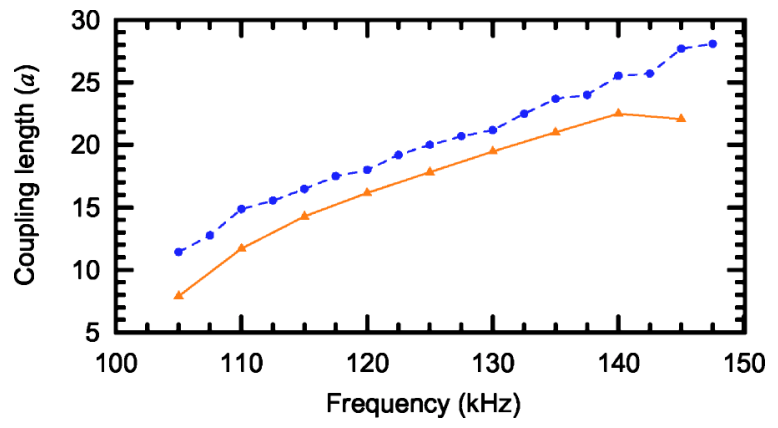

FIG. 7. (Color online) The relationship between the coupling lengths and frequency of elastic waves. The dashed line is the calculated half a beat length and the solid line is the result of FDTD numerical experiments. 
dicted coupling length by the calculation of half a beat length is represented by the dashed line in Fig. 7.

Thus, the coupling length is a function of frequency depending on the difference of the wave vectors of supermodes. The larger the difference is, the shorter the coupling length will be. The coupling length relations obtained from calculation of the beat length and FDTD numerical experiments show the same tendency. The mismatch may be caused by the input of the longitudinal wave source. Hence, the power of the symmetric and antisymmetric supermodes is not equal. The power transfer between two waveguides requires that both the symmetric and antisymmetric supermodes can propagate in the structure. In the present case, the power transfer does not occur below $100 \mathrm{kHz}$ since antisymmetric supermodes do not exist. Our analysis mainly focuses on the range $105-145 \mathrm{kHz}$, or the coupling length cannot be evaluated by the beat frequency analysis because there are too many allowed modes of the elastic wave. For example, with $f=155 \mathrm{kHz}$, it causes a larger mismatch of coupling lengths in Fig. 7 for frequencies higher than $145 \mathrm{kHz}$.

\section{ANALYSIS OF AN ELASTIC WAVE COUPLER}

The beat phenomenon of the two-waveguide system proves the elastic wave power transfer between two joined waveguides is feasible with a necessary coupling length, which varies with different frequencies. The similar coupling effect of photonic crystals has been adopted to serve as the wavelength division multiplexing (WDM) in optic communication devices. ${ }^{20}$ In phononic crystal structure, joined waveguides can potentially be employed as a power switch of the acoustic wave, which can also control the mechanical power. The concept is realized by patterning the coupler length to switch the elastic wave with a specific frequency. In the system of joined waveguides, a cross state appears when incident elastic waves switch from one waveguide into another and the coupler length is an odd multiple of the coupling length. However, the bar state ${ }^{20}$ appears when the elastic wave propagates in and out through the input waveguide, causing the coupler length to be an even multiple of the coupling length.

In Fig. 8(a), we show an elastic wave coupler with an interactive length of $33 a$ with one entrance on the left side and two exits on the right side. From the relationship shown in Fig. 7, we estimate that the coupler will perform the cross state at $109 \mathrm{kHz}$ for the elastic wave and at $121 \mathrm{kHz}$ for the bar state. Numerical experiments are shown in Figs. 8(b) and 8 (c), respectively. The displacement field of the propagating wave with $f=109 \mathrm{kHz}$ shows that the coupler demonstrates a cross state while the coupler with $f=121 \mathrm{kHz}$ acts as a bar state. It is worth noting that although the elastic wave resonates in the joint area of outlets in Fig. 8(c), the power leaves the coupler remaining in the bar state, as we predicted. To quantify the efficiency of power transfer, we analyze the displacement of each exit. Power exchange ratios between two outputs are $26 \mathrm{~dB}$ for the cross state and $28 \mathrm{~dB}$ for the bar state. Therefore, the system of two waveguides consisting of solid/solid phononic crystal can potentially be treated as an elastic wave coupler for the elastic wave in the solid media, (a)

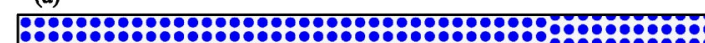

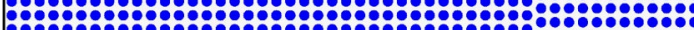

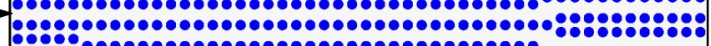
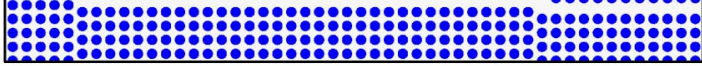

(b) $f=109 \mathrm{kHz}$

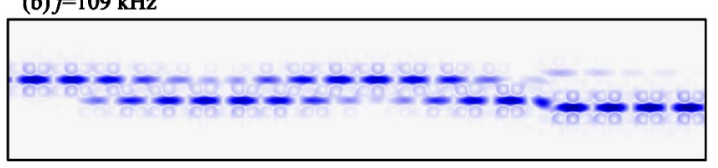

(c) $f=121 \mathrm{kHz}$

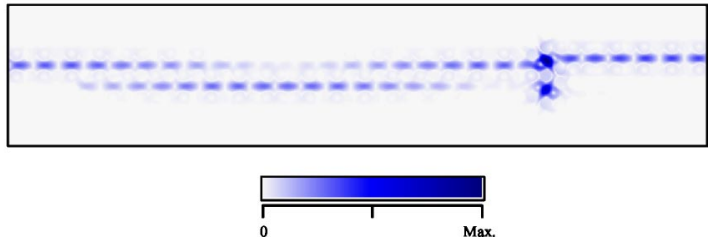

FIG. 8. An elastic wave coupler and displacement fields of propagating waves. (a) The cross section of the elastic wave coupler with a coupler length of $33 a$. (b) The amplitude of the $109 \mathrm{kHz}$ elastic wave which shows a cross state. (c) The amplitude of the $121 \mathrm{kHz}$ elastic wave which shows a bar state.

and the selection of waves with different central frequencies is controlled by the coupling length of the coupler.

Obviously, a coupler with a shorter coupling length is more flexible for the purpose of application. The previous analysis shows that the greater the coupling effect, the larger the difference of supermodes. Therefore, an enhanced system of joined parallel waveguides is proposed. The inset of Fig. 9 shows the design of the cross section of enhanced joined waveguides. The structure is similar to the waveguides in Fig. 4 except that the diameter of unit cells between the waveguides I and II, i.e., the separation of the joined parallel

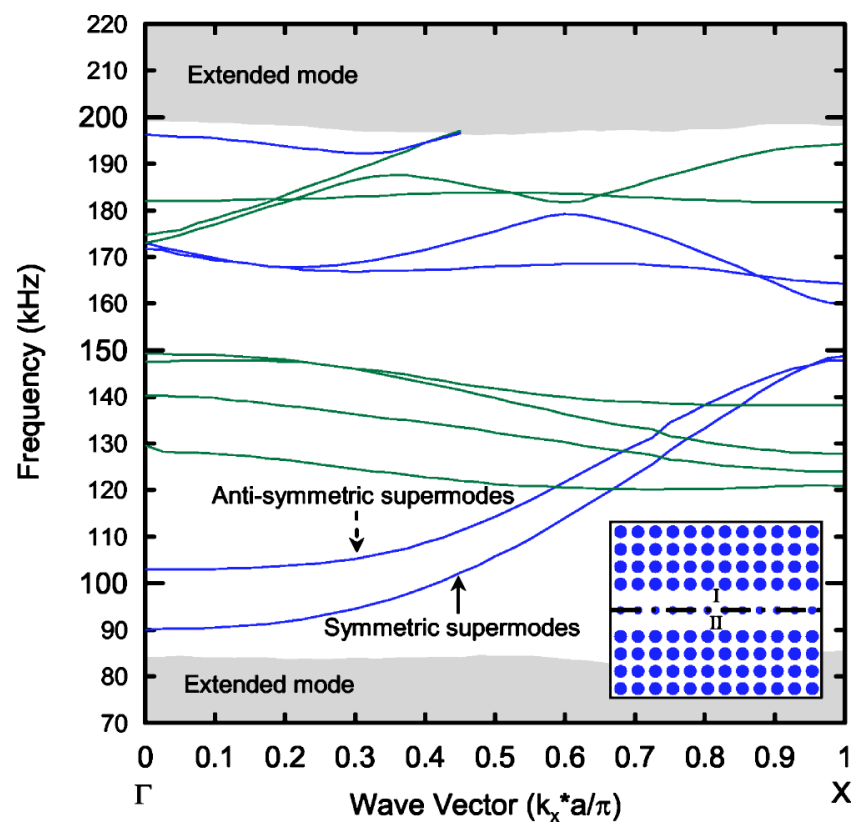

FIG. 9. (Color online) The dispersion relation of the enhanced joined parallel waveguides. 


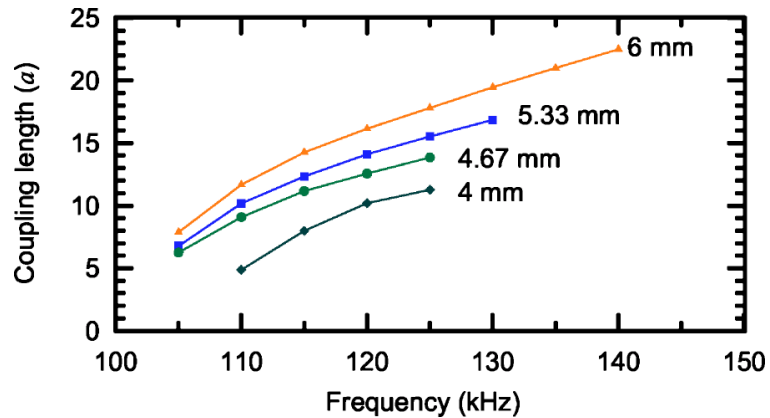

FIG. 10. (Color online) The coupling lengths of joined parallel waveguides with different separations.

waveguides, is smaller (4 $\mathrm{mm}$ in this case). Dispersion curves shown in Fig. 9 validate our enhanced design in which the variation of wave vectors for different supermodes is larger than that of the previous structure. Further, relation between the coupling length and separation $(6 \mathrm{~mm}$, $5.33 \mathrm{~mm}, 4.67 \mathrm{~mm}$, and $4 \mathrm{~mm}$ ) of the joined parallel waveguides is studied and shown in Fig. 10. The result shows that the smaller the separation, the shorter the coupling length.

To design an enhanced coupler, the enhanced joined waveguides with a separation of $4 \mathrm{~mm}$ are chosen. The length of the enhanced coupler is $20 a$, which is smaller than the standard coupler of $33 a$ with a separation of $6 \mathrm{~mm}$. The enhanced coupler performs a cross state at $112.8 \mathrm{kHz}$ and a bar state at both 119.5 and $110.2 \mathrm{kHz}$. Table I shows the comparison of the two mentioned couplers. The result shows that power exchange ratios of the enhanced coupler are smaller than those of the standard one, but still larger than $12 \mathrm{~dB}$. Obviously, with the enhanced joined waveguides, the size of the enhanced elastic wave coupler can be reduced and multiselection in the same coupler is possible.

Although the above analyses show that the bulk wave coupler proposed is promising, we note that the big diffraction loss of bulk waves has to be faced. One interesting alternative is to use surface wave propagation in a twodimensional phononic crystal. ${ }^{6}$ Detailed analysis of such a
TABLE I. Comparison of the two couplers.

\begin{tabular}{cccccc}
\hline \hline & \multicolumn{2}{c}{ Cross state } & & \multicolumn{2}{c}{ Bar state } \\
\cline { 2 - 3 } \cline { 5 - 6 } $\begin{array}{c}\text { Coupler } \\
\text { separation/length }\end{array}$ & $\begin{array}{c}\text { Frequency } \\
(\mathrm{kHz})\end{array}$ & $\begin{array}{c}\text { Power ratios } \\
(\mathrm{dB})\end{array}$ & $\begin{array}{c}\text { Frequency } \\
(\mathrm{kHz})\end{array}$ & $\begin{array}{c}\text { Power ratios } \\
(\mathrm{dB})\end{array}$ \\
\hline $6 \mathrm{~mm} / 33 a$ & 109 & 26 & & 121 & 28 \\
$4 \mathrm{~mm} / 20 a$ & 112.8 & 19 & & 119.5 & 18 \\
& & & 110.2 & 12 \\
\hline \hline
\end{tabular}

surface wave coupler awaits further study. It is worth noting that in the study of a surface wave coupler, the existing image technique ${ }^{27,28}$ can be employed to check the validity of computation results.

\section{SUMMARY}

In this paper, we have investigated the coupling phenomenon of joined parallel phononic crystal waveguides. Twodimensional steel/epoxy phononic crystal and the FDTD method are employed to study the coupling effects of joined phononic waveguides. The supermodes resulting from the coupling effect between the joined waveguides can be treated as split modes of the defect modes. We demonstrated that supermodes are indeed symmetric and antisymmetric modes which split from defect modes of a single waveguide. The result shows that the symmetric supermode shifts toward a lower frequency while the antisymmetric mode veers toward a higher frequency. A beat phenomenon between symmetric and antisymmetric modes arises when both modes are excited. The coupling lengths needed to transfer the power from one waveguide to another are evaluated by numerical experiments, and results can be interpreted by the concept of beat length. Finally, an enhanced elastic waveguide coupler is proposed and demonstrated that the coupling length can obviously be reduced.

\section{ACKNOWLEDGMENTS}

The authors acknowledge the financial support of this research by the National Science Council of ROC (NSC 922212-E-002-058) and NTU-ITRI center.
${ }^{1}$ M. S. Kushwaha, P. Halevi, L. Dobrzynski, and B. DjafariRouhani, Phys. Rev. Lett. 71, 2022 (1993).

${ }^{2}$ M. S. Kushwaha, P. Halevi, G. Martinez, L. Dobrzynski, and B. Djafari-Rouhani, Phys. Rev. B 49, 2313 (1994).

${ }^{3}$ M. S. Kushwaha and P. Halevi, Appl. Phys. Lett. 64, 1085 (1994).

${ }^{4}$ Y. Tanaka and S. I. Tamura, Phys. Rev. B 58, 7958 (1998).

${ }^{5}$ Y. Tanaka and S. I. Tamura, Phys. Rev. B 60, 13294 (1999).

${ }^{6}$ Tsung-Tsong Wu, Zi-Gui Huang, and S. Lin, Phys. Rev. B 69, 094301 (2004).

${ }^{7}$ F. R. Montero de Espinosa, E. Jimenez, and M. Torres, Phys. Rev. Lett. 80, 1208 (1998).

${ }^{8}$ M. Torres, F. R. Montero de Espinosa, D. Garcia-Pablos, and N. Garcia, Phys. Rev. Lett. 82, 3054 (1999).
${ }^{9}$ M. Torres, F. R. Montero de Espinosa, and J. L. Aragon, Phys. Rev. Lett. 86, 4282 (2001).

${ }^{10}$ R. E. Vines J. P. Wolfe, and A. V. Every, Phys. Rev. B 60, 11871 (1999).

${ }^{11}$ R. E. Vines and J. P. Wolfe, Physica B 263-264, 567 (1999).

${ }^{12}$ J. O. Vasseur, P. A. Deymier, B. Chenni, B. Djafari-Rouhani, L. Dobrzynski, and D. Prevost, Phys. Rev. Lett. 86, 3012 (2001).

${ }^{13}$ M. Kafesaki, M. M. Sigalas, and N. García, Phys. Rev. Lett. 85, 4044 (2000).

${ }^{14}$ A. Khelif, B. Djafari-Rouhani, J. O. Vasseur, P. A. Deymier, Ph. Lambin, and L. Dobrzynski, Phys. Rev. B 65, 174308 (2002).

${ }^{15}$ A. Khelif, B. Djafari-Rouhani, J. O. Vasseur, and P. A. Deymier, Phys. Rev. B 68, 024302 (2003).

${ }^{16}$ A. Khelif, A. Choujaa, B. Djafari-Rouhani, M. Wilm, S. Ballan- 
dras, and V. Laude, Phys. Rev. B 68, 214301 (2003).

${ }^{17}$ A. Khelif, B. Djafari-Rouhani, V. Laude, and M. Solal, J. Appl. Phys. 94, 12 (2003).

${ }^{18}$ J. O. Vasseur, P. A. Deymier, A. Khelif, Ph. Lambin, B. DjafariRouhani, A. Akjouj, L. Dobrzynski, N. Fettouhi, and J. Zemmouri, Phys. Rev. E 65, 056608 (2002).

${ }^{19}$ Y. Pennec, B. Djafari-Rouhani, J. O. Vasseur, A. Khelif, and P. A. Deymier, Phys. Rev. E 69, 046608 (2004).

${ }^{20}$ Stefano Boscolo, Michele Midrio, and Carlo G. Someda, IEEE J. Quantum Electron. 38, 1 (2002).

${ }^{21}$ Sergey Kuchinsky, Vladislav Y. Golyatin, Alexander Y. Kutikov, Thomas P. Pearsall, and Dusan Nedeljkovic, IEEE J. Quantum Electron. 38, 10 (2002).
${ }^{22}$ F. S.-S. Chien, Y.-J. Hsu, W.-F. Hsieh, and S.-C. Cheng, Opt. Express 12, 1119 (2004).

${ }^{23}$ Y. Tanaka, Y. Tomoyasu, and S. I. Tamura, Phys. Rev. B 62, 7387 (2000).

${ }^{24}$ J. Berenger, J. Comput. Phys. 144, 185 (1994).

${ }^{25}$ W. C. Chew and Q. H. Liu, J. Comput. Acoust. 4, 341 (1996).

${ }^{26}$ E. Hecht, Optics, 4th ed. (Addison-Wesley, San Francisco, 2002).

${ }^{27}$ R. E. Vines, Shin-ichiro Tamura, and J. P. Wolfe, Phys. Rev. Lett. 74, 2729 (1995).

${ }^{28}$ Y. Sugawara, O. B. Wright, O. Matsuda, M. Takigahira, Y. Tanaka, S. Tamura, and V. E. Gusev, Phys. Rev. Lett. 88, 185504 (2002). 